a socioecological framework, to identify facilitators and barriers of PS.

Results 77 people from 31 cities submitted a total of 92 submissions, of which 53 remained eligible. Among participants with eligible entries, $60 \%$ were male, more than half identified as homosexual or bisexual, and $11 \%$ disclosed as living with HIV. Content analysis identified novel strategies to facilitate PS, including differentiation of care and stepwise notification. In addition, people-centered principles were highlighted, as emerged themes from the submissions emphasized on index education and self-empowerment, and the necessity to provide safe and supportive disclosure services.

Conclusions The contest engaged a diverse population of participants to contribute to the development of people-centered PS for MSM living with HIV in China. Differentiation of care and stepwise notification could be valuable for the nest-step design of a more comprehensive, integrated intervention package. The emphasis on people-centered PS is also insightful for HIV-related policy design in China.

\section{P432 THE PREVALENCE OF CO-INFECTION WITH HIV, HEPATITIS C AND TB AMONG PATIENTS INFECTED BY HIVIAIDS IN SENEGAL}

P Sow*. Université De Bambey, Bambey, Sénégal

10.1136/sextrans-2021-sti.452

Hepatitis is the predominant risk factor associated with hepatocellular carcinoma.

Objective The aim of the present research is to carry out epidemiological studies concerning the hospital out-come management of Hepatitis C co - infection with HIV and TB in a well defined hospital based population. Materials and

Methods Epidemiological studies were carried out among 1320 patients admitted at Kaolack hospital concerning the hospital out-come management of hepatitis $\mathrm{C}(\mathrm{HCV})$ and Co - infections with HIV and TB over a period of four years (2006 - 2010). The grand diagnostic kits were used to analyze blood samples for HBV, HCV and HIV while sputum samples were analyzed for $\mathrm{TB}$ using the Zel Nielsen (ZN) staining technique.

Results A CD4 lymphocyte count of $0-200$ cells $/ \mathrm{mml}$ was found in $625(47.3 \%)$ individuals. Out of these, 393 were found to be in WHO stage 1 clinical status. A further 409 with CD4 count of $201-400 \mathrm{cell} / \mathrm{mml}$ were grouped as stage Ill (254) and stage IV (155). These together was determined as being eligible for anti-viral therapy. Of background Viral hepatitis constitutes a major public health problem world wide. Viral those eligible for anti-viral 52 and 80 were infected with $\mathrm{HCV}$ and HIV respectively. With respect to subjects management outcome in the hepatitis $\mathrm{C}$ infected subjects, follow-up was lost in $44(71.0 \%)$ while $11(17.7 \%)$ subjects survived and 7 (11. 3\%) died. Similarly, subjects managements out-come amongst HIV and TB co-infected with HCV infections were fallow-up was lost in $34(77.3 \%), 7$ (15.9\%) survived and 3 $(6.8 \%)$ died. With respect to TB smear positive co-infected with HCV, fallow-up was lost, 6 (20.6\%) survived while 6 (20.6\%) died.

Conclusion In respect to CD4 lymphocyte count and WHO clinical staging subjects were selected for Antiviral therapy management out-come showed that there was loss of fallowup for majority of the subjects.

\section{P436 ANALYSIS OF SOME INDICATORS OF IMMUNE STATUS AND A NUMBER OF CYTOKINES IN CHLAMYDIA INFECTION AND MYCOPLASMOSIS}

O Kukhta, M Voloshynovych*. Ivano-Frankivsk National Medical Univercity, Ivano-Frankivsk, Ukraine

\subsection{6/sextrans-2021-sti.453}

Background Among the current and common STIs are urogenital chlamydia infection and mycoplasmosis, which lead to significant complications, infertility, pathology of pregnancy and childbirth. A characteristic feature of inflammatory diseases of the genitourinary system caused by mono- and mixed infection (chlamydia and mycoplasma) is the immunological reorganization of the organism, which is confirmed by immunological methods.

Methods To study the immune status of patients with chlamydia and mycoplasmosis, clinical and laboratory tests of 64 individuals aged 17 - 36 years were performed. Control group included 6 practically healthy people. The number of total T-lymphocytes (CD3), T-helpers (CD4) and T-suppressors (CD8) was determined in all patients by indirect immunofluorescence by staining cells in a monolayer, as well as the level of a number of cytokines (IL-2; IL-3; IL-4; IL-6) by ELISA. The significance of differences between the independent values was determined using Student's t-test.

Results Objective examination revealed the following: cervical hyperemia - 48 (75\%), urethral hyperemia - 14 (21,9\%), mild cervical edema - 16 (25\%), discharge - in all patients.

Study of T-cell component of immunity revealed a significant decrease in total population of T-lymphocytes (CD3), Thelpers (CD4) and an increase in the number of cells with suppressor capacity (CD8). These changes in subpopulation ratio of T-lymphocytes lead to a significant inversion of the immunoregulatory index (CD4/CD8).

The study showed significant changes in some indicators of cytokines, in particular: a significant increase in IL-3, IL-4 and IL-6. Significant reduction in the amount of proinflammatory cytokine IL-2 was noted.

Conclusion Immune status disorders are observed in women with urogenital chlamydia infection and mycoplasmosis. In particular, changes in the population of T3 lymphocytes CD3, CD4, CD 8 and $C D 4 / C D 8$, as well as changes in the level of a number of cytokines (IL-2; IL-3; IL-4; IL-6) are revealed.

\section{P437 CULTURE AS SOCIAL DETERMINANT OF HEALTH DETERRENT TO MSM HEALTH IN KWAZULU NATAL, SOUTH AFRICA}

A lkhile*, A Mavhandu-Mudzusi. University of South Africa, Pretoria, South Africa

\subsection{6/sextrans-2021-sti.454}

Background Access to health-services for men-who-have-sexwith-men (MSM) is critical in the fight against HIV/AIDS. MSM across South-Africa are experiencing factors preventing them from accessing health-services and have been denied their fundamental human rights. Culture has been identified as one of the key barriers for MSM in accessing health services. Method The study used two data collection tools: in-depth interview and observation for 25 MSM. The interviews were conducted in Durban KZN province focusing on experiential assessments of MSM in the district municipality. The selected 